\title{
Investigation of the Effect of Pipeline Size on the Cross Flow Injection Process
}

\author{
Mohamed Elashmawy \\ Dpt of Mechanical Engineering, \\ University of Hail, Saudi Arabia and \\ Dpt of Engineering Science, Suez \\ University, Egypt \\ arafat_696@yahoo.com
}

\author{
Abdulaziz Alghamdi \\ Mechanical Engineering \\ Engineering College \\ University of Hail \\ Saudi Arabia \\ a.alghamdi@uoh.edu.sa
}

\author{
Isam Badawi \\ Mechanical Engineering \\ Engineering College \\ University of Hail \\ Saudi Arabia \\ isam149@gmail.com
}

\begin{abstract}
Injection pumps constitute an essential component for many industrial applications. The main focus of this study is to predict the effect of the size of the pipeline on the cross flow injection process. A test-rig was designed, built and equipped with three different pipelines, 11/2", 3/4" and 1/2" diameters. Comparison was made under constant line pressure of 40-bar and line flow rate of $5 \mathrm{liter} / \mathrm{min}$, with a fixed injection pump rotational speed of $100 \mathrm{rpm}$. The main parameter tested was the injection dose capacity at different pump displacements. Cross flow mixing process is also theoretically studied using 3D-CFD analysis to show the injection cross flow behavior for the same geometry and parameters used for experimental test. Results show that increasing the size of the pipeline increases injection pump doses ability. This effect is insignificant at lower injection pump displacements, while the effect of the size of the pipeline becomes dominant when increasing the displacement. By changing the size of the pipeline from $1 / 2$ " to $1 \frac{1 / 2}{2}$ diameter injection pump dose capacity increases by $3.24 \%$ at $100 \%$ pump displacement. Selecting larger pipe sizes for injection ports is recommended whenever possible.
\end{abstract}

Keywords- injection; mixing; cross-flow; CFD; receprocating

\section{INTRODUCTION}

Injection technology is essential for many industrial processes. The most widely used type is the diaphragm injection pump unit due to its simple and compact low cost design. Diaphragm pumps have many problems related to their diaphragm material and electro-magnetic core. The membrane (diaphragm) is aggressively subjected to cyclic fatigue and high stresses at certain points which cause material failure within a relatively short time period. The different behavior in pumping speed and ultimate pressure of rotational speed controlled diaphragm pumps in comparison to constant-speed pumps is related to the mechanical properties of the valves and gas dynamics [1].

Driving of the injection metering pumps could be hydraulically actuated, air/gas driven, electric/engine or power impelled. Diaphragm pumps are normally operated by a heavy-duty gear reducer system for superior performance. Hydraulically actuated diaphragm minimizes diaphragm fatigue. In case of a very low injection doses requirement micro injection pumps should be used. The parameters of the micro valveless pump were theoretically and experimentally investigated. Results provide a useful reference for structure optimization of the micro valveless pump driving diaphragm [2]. The weak component of a pneumatic diaphragm pumps is its diaphragm material which govern the pump lifetime [3]. A developed inexpensive 6-bar flow system was introduced based on a low-cost diaphragm pump [4]. In order to satisfy high pressure injection applications, a piston pump unit should be used. Piston pumps satisfy high volumetric efficiency due to a tight gap space between piston and cylinder [5].

Another application concerning the mixing process is the T-junction used for cooling systems. In [6], authors studied the turbulent jet mechanics stating an optimum tee mixing condition. The study results show the importance of using the $90^{\circ}$ bend after mixing pipe. The momentum ratio of the main and the branch velocities of the T-junction is also an important parameter. In [7], authors studied the mixing mechanism of the T-junction with the $90^{\circ}$ bend. A visualization study was performed using a test rig having three sections, for separated visualization using particle image velocimetry. The three main mixing regions were clearly visualized. The strongest region having high cycle thermal fatigue was found near the downstream of wake region. In [8], authors studied the momentum ratio for general angled T-junctions using CFD simulation. The study shows the importance of cross flow angle parameter on increasing the velocity ratio which is an important parameter to enhance the design of pipeline systems.

The main parameter in focus of the present study is the effect of the pipeline size on the performance of the injection process. An experimental test section was built of 3-different pipe sizes with a possibility to use each pipeline separately. A 3D-CFD study was also performed for the same three pipelines for comparison and evaluation of mixing process behavior based on velocity and pressure distribution within the mixing region.

\section{EXPERIMENTAL SETUP}

A high pressure water closed circuit test-rig is designed and schematically shown in Figure 1. The test-rig is equipped with three different pipelines (1/2", 3/4" and 11/2") with possibility of 
operating each pipeline separately. Injection pump used is the variable displacement reciprocating injection pump (VDRIP), App. A steel pipelines according to ANSI schedule 40 are used.

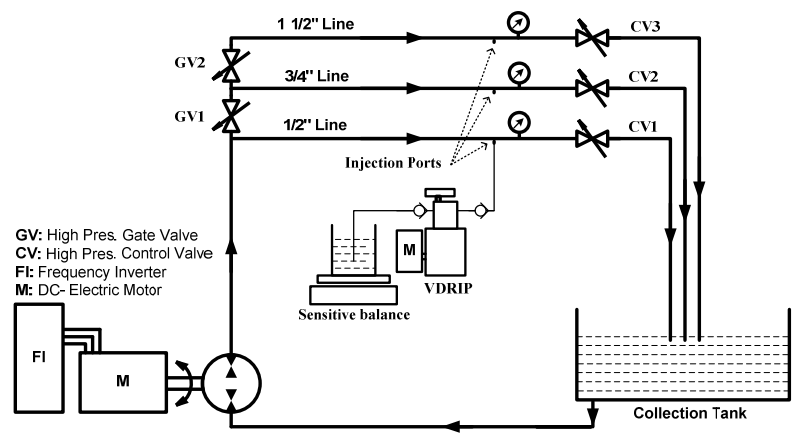

Fig. 1. Schematic diagram of the test-rig.

Each pipeline is operated separately and engaged to the test-rig by means of combination of a high pressure valves. As shown in Figure 1, there are three modes of valves:

Mode I: GV1 valve is closed;

CV1 valve controls the flow in the $1 / 2$ " line.

Mode II: GV1 valve is opened; GV2\& CV1 are closed;

CV2 controls the flow in the $3 / 4$ " line.

Mode III: GV1 and GV2 valves are opened while CV1 and CV2 are closed;

CV 3 controls the flow in the $1 \frac{1}{2}$ " line.

The injection port of the engaged line is connected to the injection pump (VDRIP) and the other two injection ports are closed using a blind stop. VDRIP is driven using a DC electric motor selected from the available automotive industry market "CHP 12V 42W" [9]. An electronic regulator with an adjustable Pulse Width Modulation (PWM) controller is used to control the speed of the VDRIP which is measured using a LASER rotational speed measuring instrument. An electric 3phase motor of $7.5 \mathrm{hp}$ with a full speed of $3400 \mathrm{rpm}$ at $60-\mathrm{Hz}$ is used. The motor speed is controlled using a $10 \mathrm{hp}$, 3-phase frequency inverter adjusted at $26.5 \mathrm{~Hz}$ output (1505 rpm, 5 liter/min).

\section{EXPERIMENTAL RESULTS AND DISCUSSION}

All experimental measurements were performed at a constant line flow rate ( 5 liter/min), constant line pressure (40bar), and constant VDRIP rotational speed (100 rpm). Figure 2 shows the effect of the size of the pipeline on the injection capacity for different pump displacements. The difference between $1 / 2$ " and $3 / 4$ " pipelines show an insignificant effect with a slight enhancement of the injection quantity for the $3 / 4$ " pipeline. Increasing the pipe diameter up to $1 \frac{1}{2} "$ enhances the injection capacity by a relatively significant percentage (3.24\% relative to $1 / 2 "$ pipeline size). The effect of the size of the pipeline becomes more significant when increasing the displacement of the injection pump. Increasing the size of the pipeline enhances the injection percentage by very small quantities for low pump displacements and becomes negligible for very low injection displacement. Significant enhancement occurs at higher displacements and maximizes at $100 \%$ pump displacement. Figure 3 shows the effect of the size of the pipeline on the injection fluid power required to drive the injection process. Increasing the size of the pipeline increases the demand of the injection fluid power at the same conditions of 40-bar pressure and $100 \mathrm{rpm}$ rotational speed. This result indicates an enhancement of injection process volumetric efficiency due to increasing the size of the pipeline. Figure 4 illustrates the effect of the size of the pipeline on the injection capacity and injection fluid power at the maximum pump displacement. The maximum increase of the injection capacity as well as injection fluid power $(3.24 \%)$ is achieved when increasing the line size from $1 / 2$ " to $1 \frac{1}{2}$ " $\left(\mathrm{A}_{\text {inj }} / \mathrm{A}_{\text {line }}\right.$ from 0.99 to $6.66)$ with $100 \%$ VDRIP displacement, while this increase is limited to $0.71 \%$ when increasing the line size from $1 / 2$ " to $3 / 4$ " $\left(\mathrm{A}_{\text {inj }} / \mathrm{A}_{\text {line }}\right.$ from 0.99 to 3.81$)$ with $100 \%$ displacement. This result indicates a significant enhancement of the volumetric efficiency of the VDRIP. Figure 5 shows the effect of the size of the pipeline on the injection capacity and the injection fluid power at $50 \%$ of pump displacement. The injection capacity as well as the injection fluid power both are increased by $1.34 \%$ when increasing the size from $1 / 2$ " to $1 \frac{1}{2}$ " $\left(\mathrm{A}_{\text {inj }} / \mathrm{A}_{\text {line }}\right.$ from 0.99 to 6.66), while this increase is limited to only $0.45 \%$ when increasing the size from $1 / 2$ " to $3 / 4 "\left(\mathrm{~A}_{\text {inj }} / \mathrm{A}_{\text {line }}\right.$ from 0.99 to 3.81$)$.

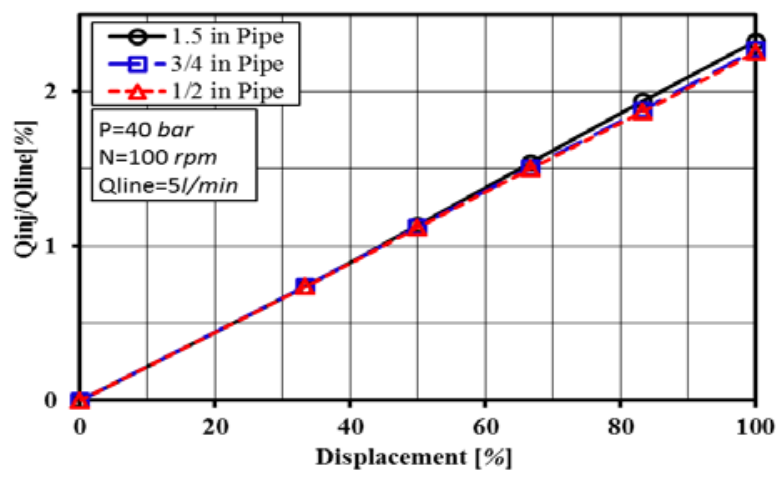

Fig. 2. Effect of the size of the pipeline on injection capacity for different VDRIP displacements.

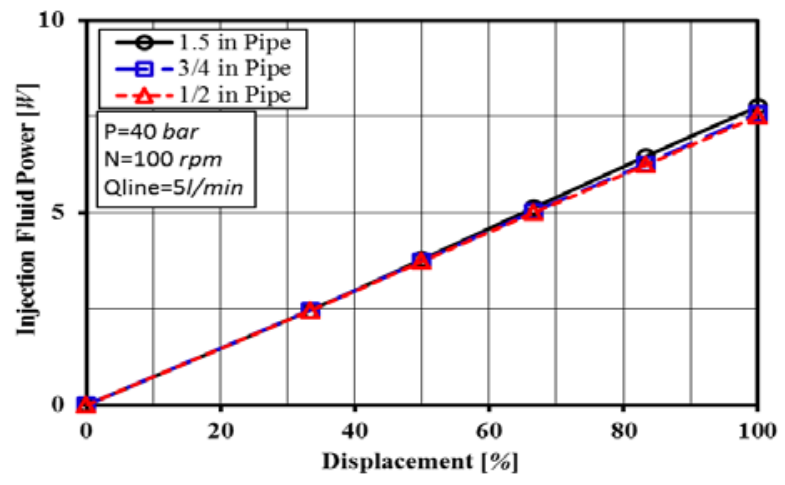

Fig. 3. Effect of the size of the pipeline on injection fluid power for different VDRIP displacements. 


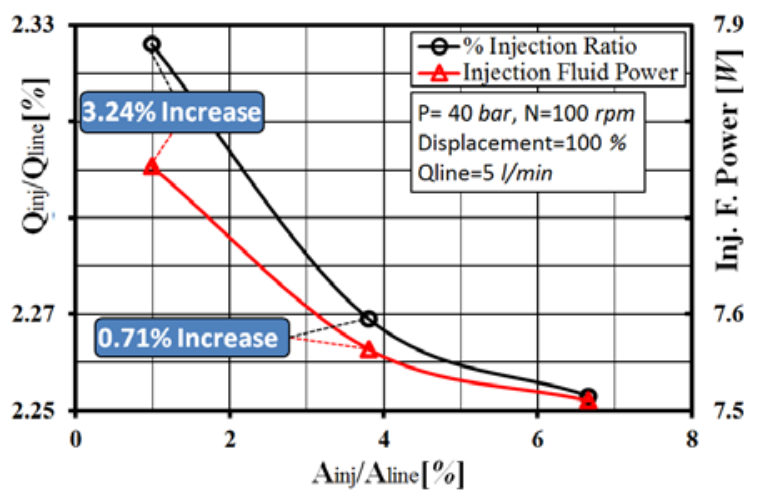

Fig. 4. Effect of the size of the pipeline on the injection capacity and the injection fluid power at $100 \%$ VDRIP displacement.

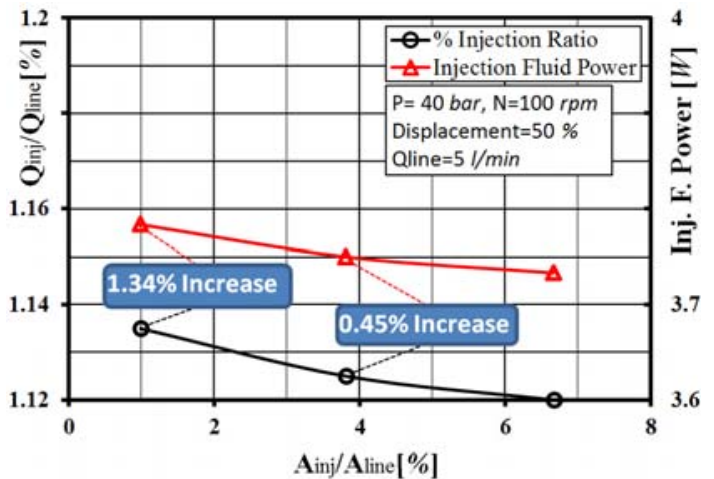

Fig. 5. Effect of the size of the pipeline on the injection capacity and the injection fluid power at 50\% VDRIP displacement.

\section{3D-CFD SIMULATION}

3D-CFD ANSYS Fluent simulation was performed using the same 3 different pipeline geometries (Figure 6). Because both line and injection streams are of water at the same initial temperatures, the mixing process is assumed to be isothermal. Pipe wall roughness is typically taken as $40 \mu \mathrm{m}$ for commercial steel pipe material. Line flow is constant ( 5 liter/min) for all line pipes. Turbulent flow regime is considered using $k-\varepsilon$ model for the three pipelines ( $1 / 2^{\prime \prime}, 3 / 4^{\prime \prime}$, and $\left.1 \frac{1 / 2}{2}\right)$ where Reynolds numbers are $\mathrm{Re}_{\mathrm{D}}=8600,6500$, and 3500 respectively. Also, injection flow is turbulent $\left(\operatorname{Re}_{\mathrm{d}}=3570\right)$. The $3 \mathrm{D}-\mathrm{CFD}$ analysis was performed for steady state taking the maximum instant injection flow as constant while in reality it was unsteady due to reciprocating single piston pump operation. The boundary conditions are: inlet mass flow rate $=0.08333 \mathrm{~kg} / \mathrm{s}$, injection mass flow rate $=0.008333 \mathrm{~kg} / \mathrm{s}$, isothermal temperature $=$ $300 \mathrm{~K}$, mixture exit pressure $=40 \mathrm{bar}$. The ANSYS Fluent default meshing was used with inflation on the pipe and injection port surfaces, the mesh size was changed and results were compared until results reach stable and symmetry contours. The maximum number of nodes and elements was 124,670 and 386,467 respectively.

Figure 7 shows the effect of the cross flow injection on the velocity distribution within the mixing region along pipe centerline (x-axis) for the three pipe sizes. Results show that increasing pipe diameter from $1 / 2$ " to $3 / 4$ " slightly enhances velocity distribution and the velocity curve tends to be flatter after mixing region. Whoever increasing pipe diameter to $1 \frac{1 / 2}{2}$ strongly enhances velocity distribution, the velocity curve is almost constant starting from short distance after mixing region. $1 \frac{1}{2}$ " pipe size satisfies the lowest velocity fluctuations after injection port which indicates best mixing conditions due to lowest wake region compared to $1 / 2$ " and $3 / 4$ " pipe sizes. Figure 8 shows the effect of the cross flow injection on the static pressure difference distribution within the mixing region along pipe centerline (x-axis) for the three pipe sizes. Increasing pipe diameter from $1 / 2$ " to $3 / 4$ " slightly enhances pressure difference distribution. Difference between maximum and minimum pressures was achieved at the injection zone and was $55 \mathrm{~Pa}$ for $1 / 2$ " pipe while reduced to $32 \mathrm{~Pa}$ for $3 / 4$ " pipe. Whoever using $1 \frac{1}{2} "$ pipe strongly enhances and reduces the pressure difference to $9.5 \mathrm{~Pa}$. After the injection port (from $\mathrm{x}=0.03$ to $0.2 \mathrm{~m}$ ), the pressure difference was $50 \mathrm{~Pa}, 17 \mathrm{~Pa}$, and $0.8 \mathrm{~Pa}$ for $1 / 2 ", 3 / 4 "$ and $1 \frac{1}{2}$ " pipes respectively. $1 \frac{1}{2}$ " pipe size satisfies the lowest pressure difference required for flow at the lowest disturbance conditions.

Figure 9 shows the stream lines of the three pipe sizes. The lower part of the figure shows separated injection stream lines in order to focus on the behavior of the injection flow streams along the mixing region. The behavior of the stream lines show that the effect of pipe size for $1 \frac{1 / 2}{2}$ pipe injection stream lines is limited in narrow distance after injection port. While for $1 / 2$ " and $3 / 4 "$ pipes takes much longer distance to get fully mixed.

Figure 10 shows the velocity contours of the three pipe sizes. The upper contours show a velocity contours of the $x-y$ plane at the centerline of the pipe $(z=0)$ which certify the results achieved by stream lines insight. Injection effect of $1 / 2$ " pipe is the strongest one and takes the longest distance before achieving complete mixing conditions. The $3 / 4 "$ pipe takes a slightly shorter distance, whoever $1 \frac{1}{2}$ " pipe takes a very short distance to get fully mixed. The three lower contours show the velocity contours of the $\mathrm{z}$-x plane at three different distances from centerline $(\mathrm{y}=\mathrm{r}=0, \mathrm{y} \approx 1 / 3 \mathrm{R}$, and $\mathrm{y} \approx 2 / 3 \mathrm{R}$ where $\mathrm{R}$ denotes the pipe radius). The $\mathrm{z}-\mathrm{x}$ plane contours show clearly the wake region downstream the injection zone, the largest wake zone is achieved for the $1 / 2$ " pipe and then the $3 / 4$ " pipe while the lowest wake zone is obtained for the $1 \frac{1}{2} "$ pipe. The results obtained by CFD analysis are consistent with the results obtained by other researchers, [7-8].

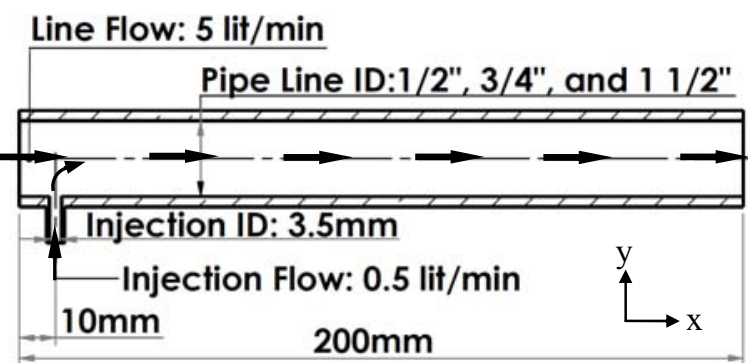

Fig. 6. Schematic diagram of the 3D-CDF test-section 

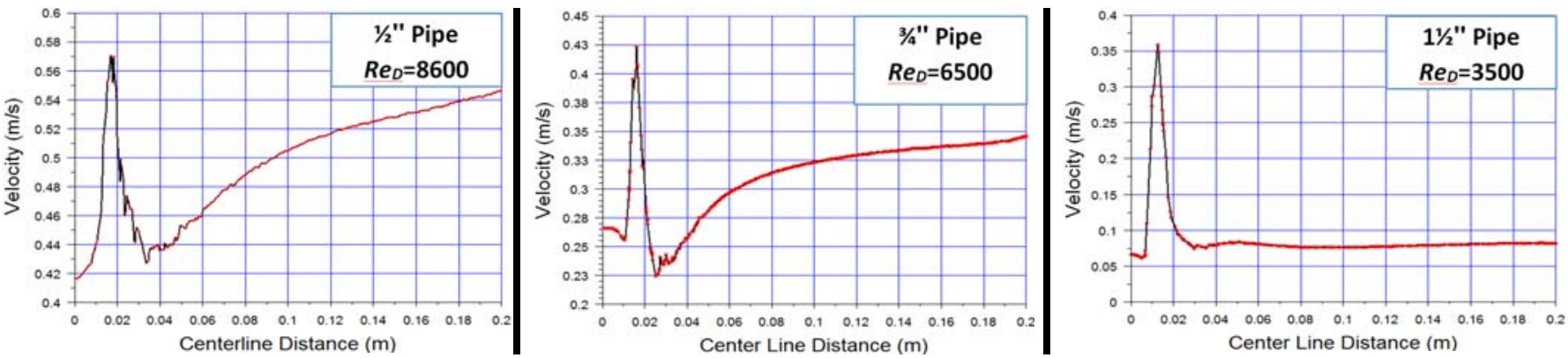

Fig. 7. Effect of cross flow injection on the line velocity magnitude along x-axis, 3D-CFD ANSYS Fluent analyses
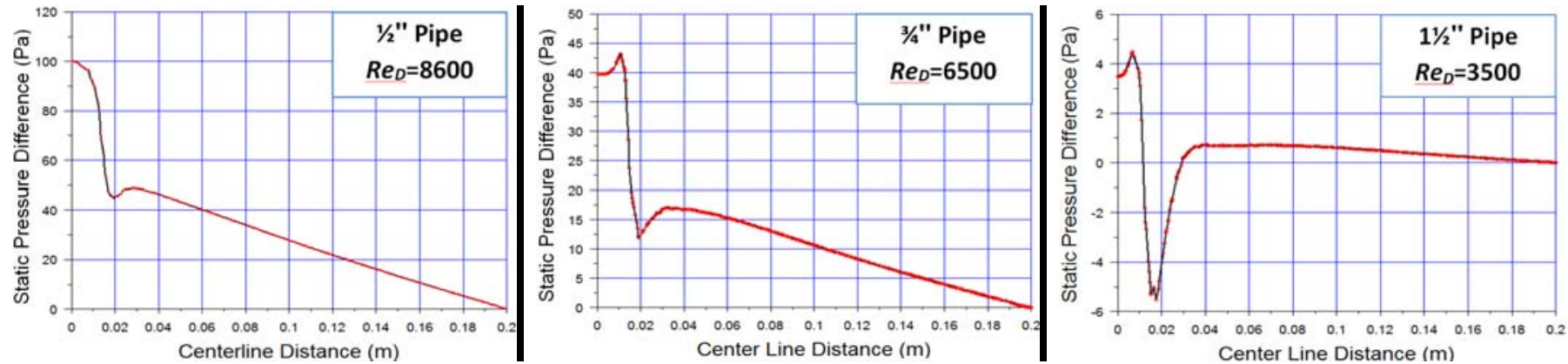

Fig. 8. Effect of cross flow injection on the line pressure difference distribution along x-axis, 3D-CFD ANSYS Fluent analyses
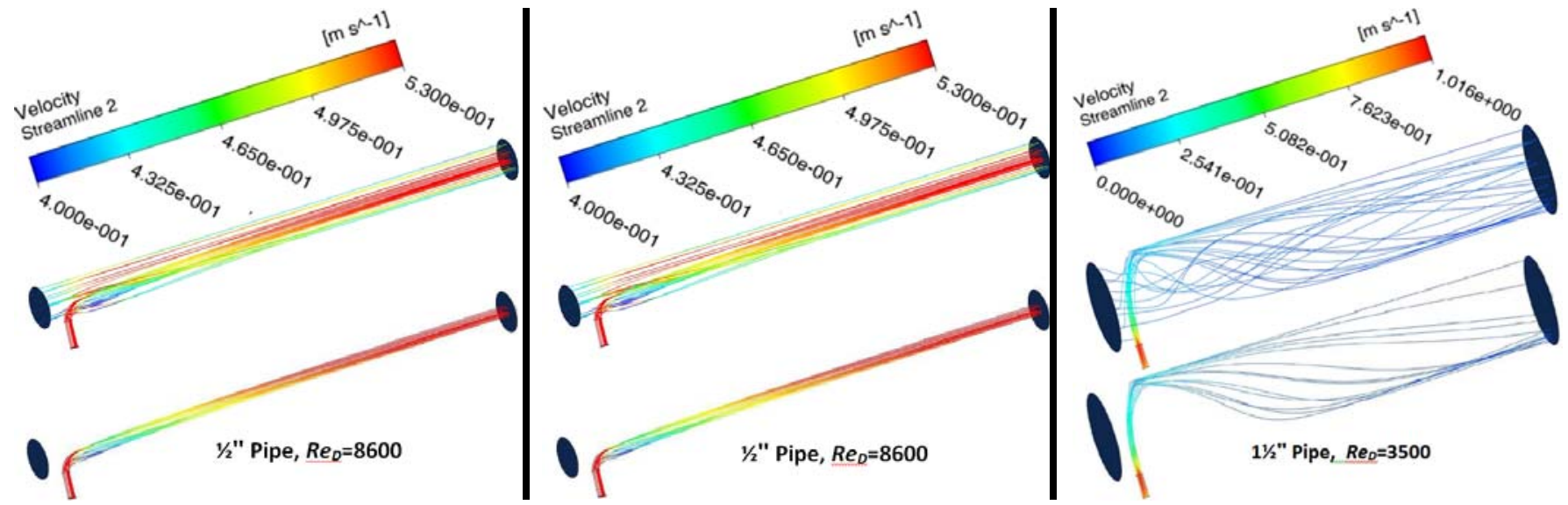

Fig. 9. Stream lines including cross flow injection, 3D-CFD ANSYS Fluent analyses

\section{CONCLUSIONS}

Both experimental and theoretical operating conditions were the same. Results are limited to 40 bar line pressure due to safety precautions $\left(1 \frac{1}{2} "\right.$ pipeline was of welded steel with ANSI schedule 40). The limitation of the pressure has no effect on the comparison results because all measurements and CFD ANSYS Fluent analysis for all lines were operated under the same pressure (40 bar). Moreover constant rotational speed (100 rpm) and constant line flow rate $(5 \mathrm{lit} / \mathrm{min})$ are fixed throughout the study. Both experimental and theoretical results show that increasing the line size has positive impact on the performance of the cross flow injection process especially at higher pump displacements with significant increase of pipeline sizes. A maximum injection enhancement of $3.24 \%$ is achieved when increasing the injected pipeline size from $1 / 2$ " to $1 \frac{1}{2} "$.

The authors recommend designers to choose injection ports at maximum line sizes positions whenever possible.

Further research efforts are also recommended especially for the following parameters:

- expanding the test for higher pressure ranges

- studying the effect of line pressure on the performance of the injection process

- studying the effect of VDRIP displacement on pump volumetric efficiency especially at low displacements to give exact lower displacement limitation for VDRIP. 


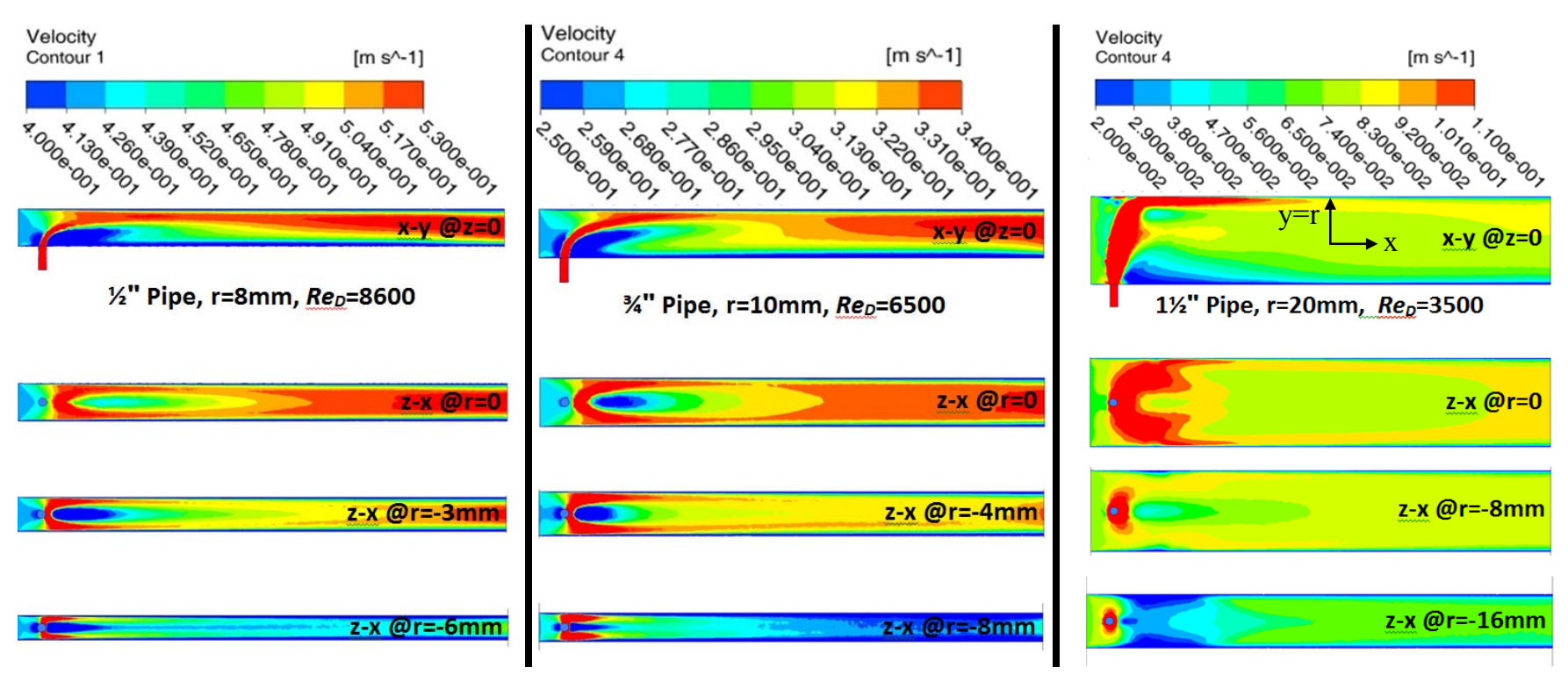

Fig. 10. Velocity contours of the cross flow mixing region for the three pipe sizes, 3D-CFD ANSYS Fluent analyses

\section{APPENDIX A- VDRIP DESIGN ASPECTS}

Figure 11 shows a schematic diagram of the VDRIP operating principal, the variable displacement is accomplished by means of a simple mechanical controlled stopper facility. The location of the stopper is driven and controlled by a threaded spindle having a hand-wheel attached to its upper end. Controlling displacement is occurred during suction stroke. When the piston reaches the stopper liver it will stop leaving the cam to complete its journey without contacting the piston end. The position where the stopper located will control the suction dose and keep it until the cam hits the piston end again at the start of the delivery stroke and force the dose trapped inside cylinder to be injected to the line. This design requires low rotational speed (satisfied by nature) to avoid serious damages due to cam piston end collisions. Figure 12 shows detailed 3D-CAD design of the VDRIP $(A)$ and a photograph of the realized VDRIP $(B)$.

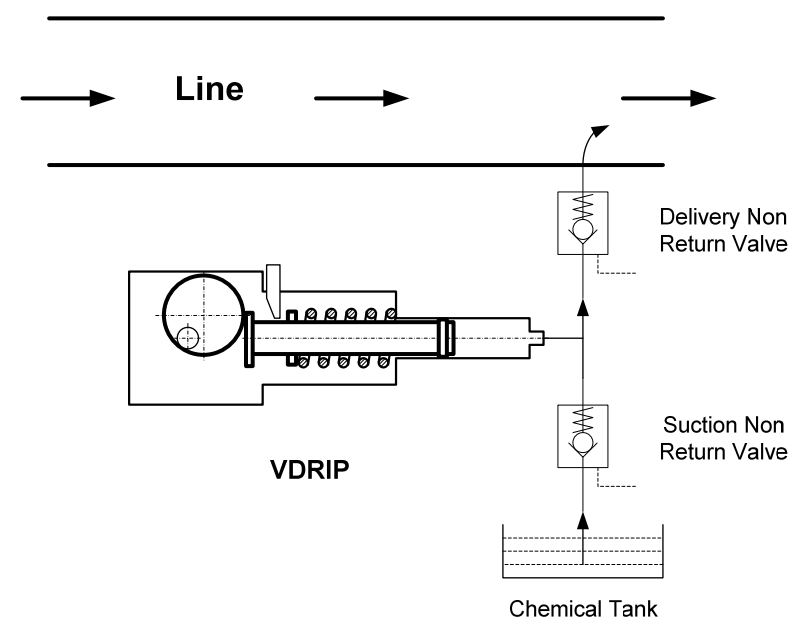

Fig. 11. Schematic diagram of the VDRIP principal.

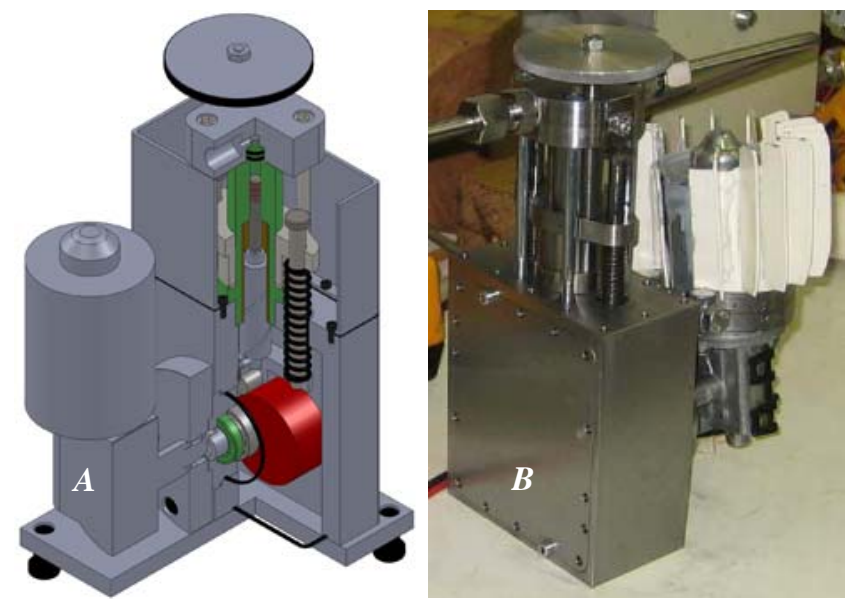

Fig. 12. A:3D-CAD of the VDRIP, B: Photograph of the realized VDRIP

\section{ACKNOWLEDGMENT}

Acknowledgments to Deanship of Scientific Research, University of Hail, KSA, for funding and supporting this project with code number of "E5-ME, 2014" and making it feasible.

\section{REFERENCES}

[1] R. Lachenmann, J. Dirscherl, "Advanced performance of small diaphragm vacuum pumps through the use of mechatronics", Applied Physics A-Material Science \& Processing, Vol. 78, pp. 671-673, 2004

[2] X. Hai-bo, F. Xin, Y. Hua-yong, "Effects of structural parameters and rigidity of driving diaphragm on flow characteristics of micro valveless pump", Journal of Zhejiang University SCIENCE, Vol. 4 No. 1, pp. 5357,2003

[3] A. Kurteev, "Diaphragms for pneumatic pumps, Chemical and Petroleum Engineering", Vol. 47, No. 7-8, pp. 550-556, 2011

[4] A. Ratka, H. Berndt, "A novel analytical low-cost flow system based on a $0.6 \mathrm{MPa}(84 \mathrm{psi})$ diaphragm pump applied to on-line trace pre- 
concentration in flame AAS and ICP-OES", Anal Bioanal Chem, Vol. 375, No. 2, pp. 275-280, 2002

[5] H. Murrenhoff, Grundlagen der fluidtechnik teil 1: Hyraulik. Umdruck zur Vorlesung, Shaker Verlag GmbH, Germany, 2005

[6] S. M. Hosseini, K. Yuki, H. Hashizume, "Classification of Turbulent Jets in a T-Junction Area With a 90-deg Bend Upstream”, Int. J. Heat Mass Transfer, Vol. 51, No. 9-10, pp. 2444-2454, 2008

[7] S. M. Hosseini, K. Yuki, H. Hashizume, "Experimental Investigation of Flow Field Structure in Mixing Tee", ASME J. Fluids Eng., Vol. 131, No. 5, p. 051103, 2009

[8] S. Qian, J. Frith, N. Kasahara, "Classification of Flow Patterns in Angled T-Junctions for the Evaluation of High Cycle Thermal Fatigue", ASME J. Pressure Vessel Technol., Vol. 137, No. 2, p. 021301, 2014

[9] Bosch, CHP 12V 42W DC motor, Data sheet, 2016, URL:http://www.bosch-ibusiness.com/boaaelmoocs/category/CHP/283/ product/ 681 